\title{
Escitalopram oxalate inhibits proliferation and migration and induces apoptosis in non-small cell lung cancer cells
}

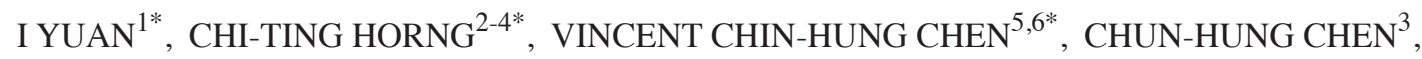 \\ LI-JENG CHEN ${ }^{3}$, TSAI-CHING HSU ${ }^{3,7}$ and BOR-SHOW TZANG ${ }^{3,7,8}$
}

\author{
Departments of ${ }^{1}$ Pharmacy and ${ }^{2}$ Ophthalmology, Kaohsiung Armed Forces General Hospital, Kaohsiung 80284; \\ ${ }^{3}$ Institute of Biochemistry, Microbiology and Immunology, Chung Shan Medical University, Taichung 40201; \\ ${ }^{4}$ Department of Pharmacy, Tajen University, Pingtung 90741; ${ }^{5}$ Department of Psychiatry, Chang Gung University, \\ Taoyuan 33302; ${ }^{6}$ Department of Psychiatry, Chiayi Chang Gung Memorial Hospital, Chiayi 61363; \\ ${ }^{7}$ Clinical Laboratory, Chung Shan Medical University Hospital; ${ }^{8}$ Department of Biochemistry, \\ School of Medicine, Chung Shan Medical University, Taichung 40201, Taiwan, R.O.C.
}

Received March 1, 2017; Accepted December 4, 2017

DOI: $10.3892 / \mathrm{ol} .2017 .7687$

\begin{abstract}
Population-based cohort studies have revealed that neuroleptic medications are associated with a reduced cancer risk. Recent studies have demonstrated that selective serotonin reuptake inhibitors (SSRIs) have an antiproliferative or cytotoxic effect on certain cancer types. Known as a superior SSRI, escitalopram oxalate exhibits favorable tolerability with generally mild and temporary adverse events. The present study aimed to examine the effects of escitalopram oxalate on non-small cell lung cancer (NSCLC) cells. The experimental results revealed that escitalopram oxalate significantly inhibited the proliferation and invasion of A549, and H460 cells compared with BEAS-2B cells. Additionally, escitalopram oxalate significantly increased the sub- $G_{1}$ population and caspase-3 activity of A549, and H460 cells. Furthermore, escitalopram oxalate significantly induced mitochondria-dependent apoptotic signaling cascades in A549 and $\mathrm{H} 460$ cells, which included increases in the protein expression levels of apoptosis regulator Bax, truncated BH3-interacting domain death agonist, cytochrome $c$, apoptotic protease-activating factor 1 , and cleaved caspase-9. These findings suggest
\end{abstract}

Correspondence to: Dr Tsai-Ching Hsu, Institute of Biochemistry, Microbiology and Immunology, Chung Shan Medical University, 110, Section 1, Jianguo North Road, Taichung 40201, Taiwan, R.O.C. E-mail: htc@csmu.edu.tw

Dr Bor-Show Tzang, Department of Biochemistry, School of Medicine, Chung Shan Medical University, 110, Section 1, Jianguo North Road, Taichung 40201, Taiwan, R.O.C.

E-mail: bstzang@csmu.edu.tw

\section{${ }^{*}$ Contributed equally}

Key words: escitalopram oxalate, non-small cell lung cancer, proliferation, migration, apoptosis that escitalopram oxalate could serve a therapeutic agent for the treatment of NSCLC due to its antiproliferative and apoptotic effects.

\section{Introduction}

Many statistical reports indicated that malignant tumors that are associated with lung cancer are the leading cause of death globally $(1,2)$. Based on its biological characteristics and clinical manifestations, lung cancer can be classified into small cell lung cancer (SCLC) and non-small cell lung cancer (NSCLC) (3). Small-cell lung tumors undergo metastasis more rapidly than NSCLC to other organs, such as the brain, skeleton, and lymph tissues (4). NSCLC can be divided into adenocarcinoma, squamous cell carcinoma, and large cell carcinoma. Large cell carcinoma is the most difficult to treat owing to its potential presence in any part of the lungs as well as its rapid growth and migration (5).

Antidepressants are frequently dispensed to cancer patients who are suffering from depressive disorders that develop in later stages. A population-based cohort study found that neuroleptic medications are associated with a reduced cancer risk (6), including a reduced lung cancer (7). An increasing number of retrospective studies are finding that selective serotonin reuptake inhibitors (SSRIs) have an anti-proliferative or cytotoxic effect on various cancers (8). Fluoxetine, a commonly used SSRI, has been demonstrated to slow the cell cycle progression in A549 cells by reducing cyclin D1 and cyclin A expressions and inducing P53 and P21 protein expressions (9). These findings reveal that antidepressants such as SSRIs may have therapeutic potential against NSCLC.

Escitalopram oxalate, also known as Cipralex ${ }^{\circledR}$ (H. Lundbeck A/S, Copenhagen, Denmark) and Lexapro ${ }^{\circledR}$ (Forest Laboratories, Inc., St. Louis, MO, USA), is a SSRI that is used for the treatment of major depressive disorder (MDD) and anxiety disorder by selectively binding to the human serotonin transporter (10). Based on a meta-analysis and a pooled analysis, escitalopram oxalate is more effective than other 
antidepressants (11). The relevant results indicated that escitalopram oxalate was superior to a placebo, and almost as effective as, or superior to, other SSRIs, including citalopram, paroxetine, fluoxetine and sertraline, and serotonin-noradrenaline reuptake inhibitors, including duloxetine and sustained-release venlafaxine. Escitalopram oxalate also exhibits favorable tolerability and causes generally mild and temporary adverse events (11). Since few studies of reports on the effects of escitalopram oxalate in NSCLC cells have been published, the present study attempts to examine the effects of escitalopram oxalate on NSCLC and the underlying mechanism involved.

\section{Materials and methods}

Cell line and escitalopram oxalate. NSCLC cell lines, A549 and H460, purchased from Taiwan Food Industry Development Research Institute (Hsinchu, Taiwan) were cultured in RPMI-1640 medium containing 5\% FBS (Gibco; Life Technologies Co., Grand Island, NY, USA). Human bronchus epithelial cell line BEAS-2B, kindly provided by Dr Gow-Tarng Sheu (Graduate Institute of Medicine, Chung Shan Medical University, Taichung, Taiwan) was cultured in RPMI-1640 medium containing 10\% FBS as control cell line. The SSRI, escitalopram oxalate (Lexapro; Sigma, St. Louis, MO, USA), was provided by Chiayi Chang Gung Memorial Hospital.

MTT assay. A total of $5 \times 10^{3}$ cells was seeded in each well of a 96-well plate and cultured overnight for cell adhesion. The culture medium was then removed and replaced with medium containing different concentrations of escitalopram oxalate. Triplicate treatments were conducted for each concentration. After incubation with different concentrations of escitalopram oxalate for 24 or $48 \mathrm{~h}$, the culture medium was removed and $0.2 \mathrm{ml} \mathrm{MTT} \mathrm{reagent}(0.5 \mathrm{mg} / \mathrm{ml})$ was added to each well for another $2 \mathrm{~h}$. A $0.2 \mathrm{ml}$ DMSO was then added to each well of the plate to dissolve the crystal and absorbance was measured at $570 \mathrm{~nm}$ with an ELISA reader. The relative cell survival rate was calculated based on the ratio of the absorbance of the sample treatment relative to the absorbance of the control treatment.

Cell-migration assay. To measure the effects of escitalopram oxalate, a modified Boyden chamber assay using cell culture inserts with a $12-\mu \mathrm{m}$ pore size polycarbonate filter in a 48 -well format was used to perform an in vitro migration assay. Cells were seeded on the upper part of the chamber at a density of $2 \times 10^{4}$ cells/well in $50 \mu \mathrm{l}$ of serum free medium. For the invasion assay, $10 \mu \mathrm{l}$ Matrigel (BD Biosciences, Bedford, MA, USA) was applied to $12-\mu \mathrm{m}$-pore size polycarbonate membrane filters, with the bottom chamber of the apparatus containing standard medium and then incubated for $16 \mathrm{~h}$ at $37^{\circ} \mathrm{C}$. The cells that had invaded to the lower surface of the membrane were fixed with methanol, washed with dd- $\mathrm{H}_{2} \mathrm{O}$, and then stained with Giemsa. Ten random fields were counted for each experiment under a light microscope at x200 magnification per filter.

Flow cytometric analysis. A total number of $2 \times 10^{6}$ cells per $100 \mathrm{~mm}^{2}$ were seeded in culture plates for $24 \mathrm{~h}$ at $37^{\circ} \mathrm{C}$ in a
$5 \% \mathrm{CO}_{2}$ incubator. The cells were then incubated with various concentrations of escitalopram oxalate for $24 \mathrm{~h}$. After incubation, the cells were harvested, washed with PBS, and fixed with $70 \%$ alcohol for $16 \mathrm{~h}$ at $4^{\circ} \mathrm{C}$. The cells were then washed using PBS and transferred into $12 \times 75 \mathrm{~mm}$ tubes. A total of $10 \mu \mathrm{l}$ of propidium iodide (PI) staining solution was added to each tube, and the contents were gently mixed. The mixture was incubated in an ice bath in the dark. Following filtration through a $40 \mu \mathrm{m}$ nylon screen, the stained cells were analyzed using a FACSCalibur analyzer (Becton Dickinson, Bedford, MA, USA).

Caspase-3 activity assay. Analysis of caspase-3 activity was performed in triplicate using the caspase-3, active form, ELISA pair kit (BD Biosciences, San Diego, CA, USA) according to the manufacturer's protocol.

Protein extraction and western blotting. Cell lysates were obtained by homogenizing the cells in lysis buffer $(20 \mathrm{mM}$ Tris-HCl, $150 \mathrm{mM} \mathrm{NaCl}, 1 \mathrm{mM} \mathrm{Na}{ }_{2}$ EDTA, $1 \mathrm{mM}$ EGTA, $1 \%$ Triton $\mathrm{X}-100,2.5 \mathrm{mM}$ sodium pyrophosphate, $1 \mathrm{mM}$ $\beta$-glycerophosphate, $1 \mathrm{mM} \mathrm{Na} \mathrm{VO}_{4}$ and $1 \mu \mathrm{g} / \mathrm{ml}$ leupeptin). The homogenates were then centrifuged at $12,000 \mathrm{x} g$ for $40 \mathrm{~min}$ and the supernatants were collected and stored at $-80^{\circ} \mathrm{C}$ for further experiments. Western blotting was performed as described elsewhere (12). Protein samples were denatured for $10 \mathrm{~min}$ in boiling water with sample buffer $(0.0625 \mathrm{M}$ Tris- $\mathrm{HCl}$ buffer, $\mathrm{pH}$ 6.8, containing 2.3\% SDS, 5\% 2-mercaptoethanol, and $10 \%$ glycerol). Samples were applied to a $12.5 \%$ Sodium dodecyl sulfate-polyacrylamide gel (SDS-PAGE) and electrophoresis at 100-150 $\mathrm{V}$ for $1.5 \mathrm{~h}$ and then electrophoretically transferred to a nitrocellulose membrane (Amersham Biosciences, Piscataway, NJ, USA). The membrane was then soaked in PBS with 5\% nonfat dry milk for $30 \mathrm{~min}$ at room temperature. Antibodies against Bax, tBid, cytochrome $c$, Apaf-1, cleaved caspase-9, phosphorylated IкB- $\alpha$ (p-IкB- $\alpha$ ) and $N F-\kappa B(p-p 65)$ and $\beta$-actin (Upstates, Charlottesville, VA, USA; Santa Cruz Biotechnology, Santa Cruz, CA, USA) were diluted in PBS with $2.5 \%$ BSA and incubated for $1.5 \mathrm{~h}$ with gentle agitation at room temperature. The membranes were washed twice with PBS-Tween for $1 \mathrm{~h}$, and a secondary antibody conjugated with horseradish peroxidase (HRP) was added. Pierce's SuperSignal West Dura HRP Detection kit (Pierce Biotechnology Inc., Rockford, IL, USA) was used to detect antigen-antibody complexes. The blots were scanned and quantified by densitometry (Appraise, Beckman-Coulter, Brea, CA, USA).

Statistical analysis. All statistical analyses were performed using SPSS 10.0 software (SPSS Inc., Chicago, IL, USA). Three independent experiments were repeated. Statistical analyses were performed using the analysis of variance plus posterior multiple comparison test to determine the difference. $\mathrm{P}<0.05$ was considered statistically significant. The significant differences were stressed with symbols as shown in figures.

\section{Results}

Escitalopram oxalate attenuates proliferation and invasive ability in A549 and H460 cells. To evaluate the effects of 

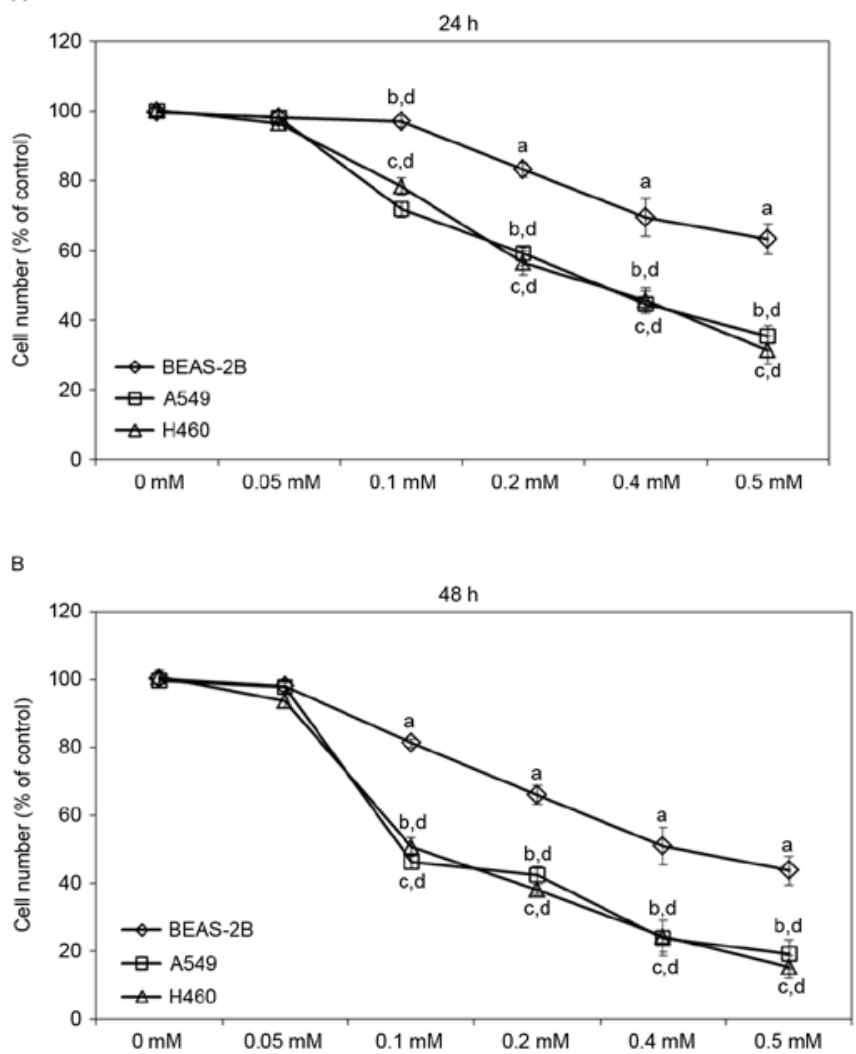

Figure 1. The effects of escitalopram oxalate on viability of BEAS-2B A549 and H460 cells. Relative survival of BEAS-2B, A549 and H460 cells treated with different concentrations of escitalopram oxalate for (A) 24 or (B) $48 \mathrm{~h}$ were determined by MTT assay. The characters, a, b and c, indicate significant difference, $\mathrm{P}<0.05$, as compared to the control group $(0 \mathrm{mM})$, and d indicates significant difference as compared to BEAS-2B cells. Similar results were observed in three repeated experiments.

escitalopram oxalate on NSCLC, A549 and H460 cell lines were treated with various concentrations of escitalopram oxalate. Treatment with $0.1,0.2,0.4$ or $0.5 \mathrm{mM}$ escitalopram oxalate for $24 \mathrm{~h}$ significantly reduced the viability of both A549 and H460 cells relative to those in the control group and the control cell line, BEAS-2B, respectively (Fig. 1A). Significantly reduced cell viability was also detected in A549 and H460 cells that were treated with $0.1,0.2,0.4$ or $0.5 \mathrm{mM}$ escitalopram oxalate for $48 \mathrm{~h}$ relative to those in the control group and the control cell line, BEAS-2B, respectively (Fig. 1B). Moreover, the invasive ability of BEAS-2B, A549 and H460 cells was evaluated as the number of cells that passed through a polycarbonate filter with $12 \mu \mathrm{m}$ pores, the number of invaded in BEAS-2B, A549 and H460 cells that were treated with $0.05,0.1,0.2,0.4$ or $0.5 \mathrm{mM}$ escitalopram oxalate for $24 \mathrm{~h}$ cells was significantly smaller than the corresponding number in the control group (Fig. 2).

Escitalopram oxalate increases sub-G1 population and induces apoptosis in A549 and H460 cells. To study the cell death pathway that is involved, BEAS-2B, A549 and H460 cells were treated with various concentrations of escitalopram oxalate for $24 \mathrm{~h}$ and examined by flow cytometry with PI staining and caspase- 3 activity assay. Significantly increased sub-G1 proportions were detected in both A549 and H460
A

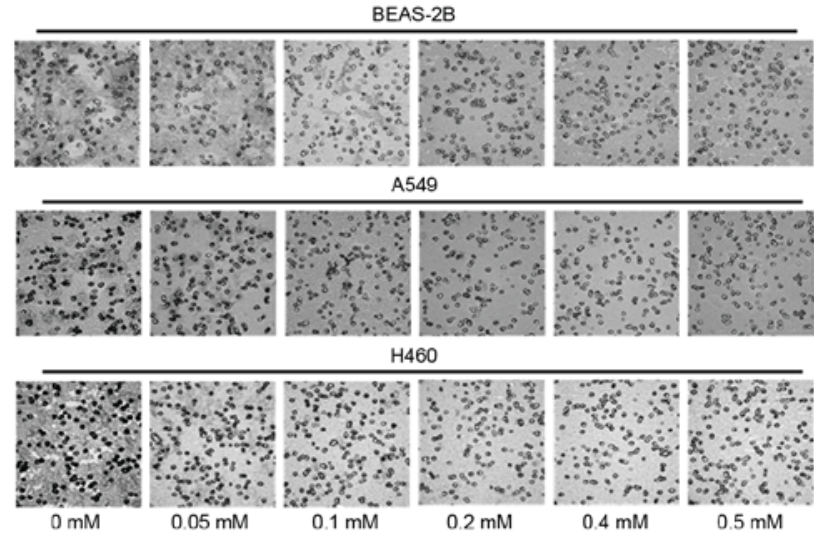

B

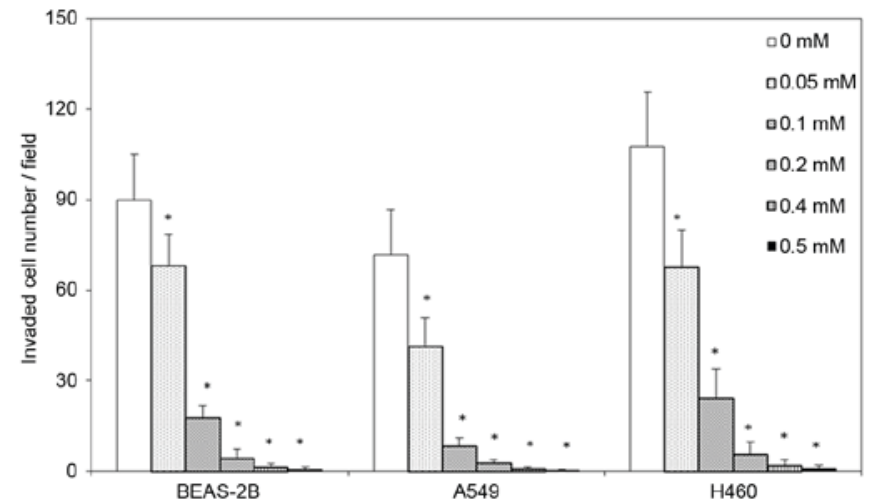

Figure 2. The effects of escitalopram oxalate on invasive ability of A549 and H460 cells after treatment with escitalopram oxalate for $24 \mathrm{~h}$. (A) Photographic pictures of invaded A549 and H460 cells that were treated with different concentrations of escitalopram oxalate (200x magnification). (B) Number of invaded cells relative to the control group $(0 \mathrm{mM})$. ${ }^{*} \mathrm{P}<0.05$ was considered to indicate a significant difference, as compared to the control group. Similar results were observed in three repeated experiments.

cells that had been treated with $0.1,0.2$ and $0.4 \mathrm{mM}$ escitalopram oxalate, relative to those in the control group or the control cell line, BEAS-2B (Fig. 3A). Significantly increased caspase- 3 activities were detected in both A549 and H460 cells that had been treated with $0.1,0.2$ and $0.4 \mathrm{mM}$ escitalopram oxalate, relative to cells in the control group or the control cell line, BEAS-2B (Fig. 3B). Since significantly increased sub-G1 and caspase-3 activity were detected in A549 and H460 cells, relative to BEAS-2B cells, the following experiments were performed on A549 and H460 cells.

Escitalopram oxalate-induced mitochondrial dependent apoptosis in A549 and H460 cells. To clarify further the signaling that is involved in escitalopram oxalate-induced apoptosis in both A549 and H460 cells, the expressions of Bax, tBid, cytochrome $c$, Apaf-1 and cleaved caspase-9 proteins were detected. Significant increases in levels of Bax and tBid proteins were detected in A549 cells that were treated with 0.2 and $0.4 \mathrm{mM}$ escitalopram oxalate for $24 \mathrm{~h}$ relative to those in the control group (Fig. 4). Thersefore, significant increases in cytochrome $\mathrm{c}$ and Apaf-1 were detected in A549 cells that were treated with 0.2 and $0.4 \mathrm{mM}$ escitalopram oxalate for $24 \mathrm{~h}$, relative to those in the control group (Fig. 4). 
A

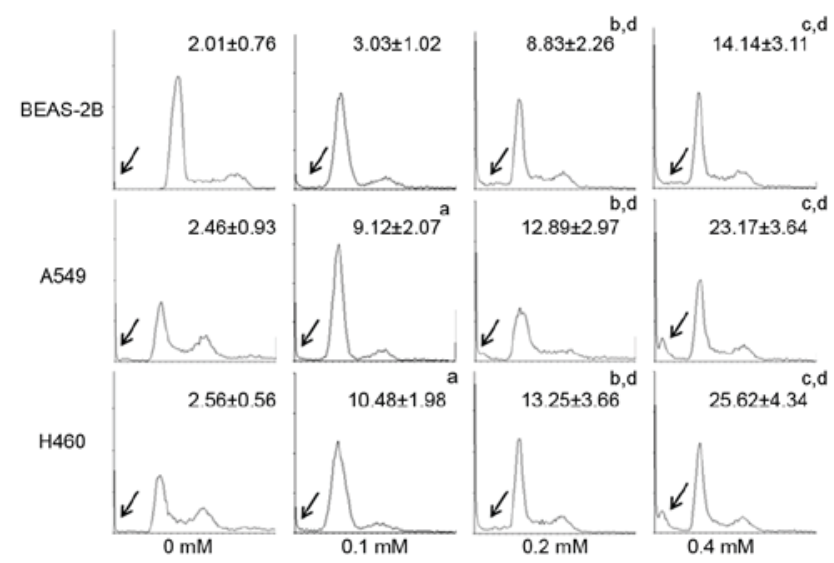

B

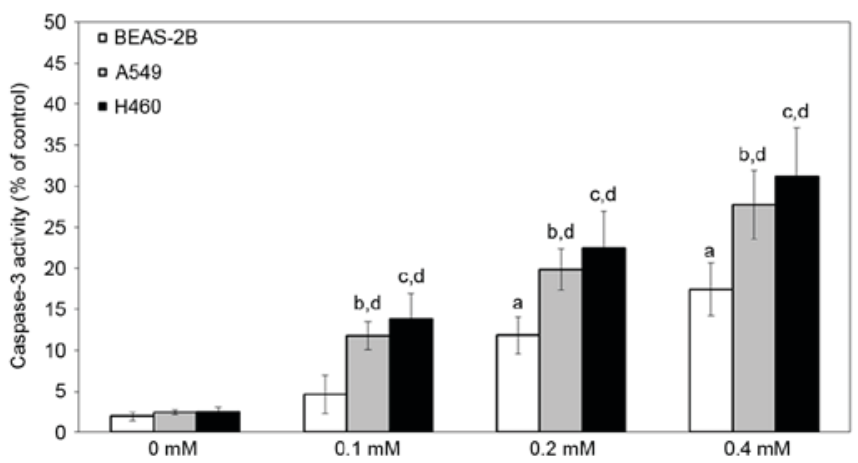

Figure 3. Escitalopram oxalate increases sub-G1 population and apoptosis in A549 and H460 cells. (A) Representative results of flow cytometry with PI staining, arrow indicated the sub-G1 proportion and (B) caspase-3 activity in A549 and H460 cells that were treated with different concentrations of escitalopram oxalate for $24 \mathrm{~h}$. The characters, a, b and c, indicate significant difference, $\mathrm{P}<0.05$, as compared to the control group $(0 \mathrm{mM})$, and indicates significant difference as compared to BEAS-2B cells. Similar results were observed in three repeated experiments.

Furthermore, cleaved caspase-9, the level of a downstream molecule of Apaf-1, was significantly increased in A549 cells that were treated with 0.2 and $0.4 \mathrm{mM}$ escitalopram oxalate for $24 \mathrm{~h}$, relative to those in the control group (Fig. 4). Similar results were observed for H460 cells. Significantly increased Bax, tBid, cytochrome $c$, Apaf-1 and cleaved caspase- 9 protein levels were detected in $\mathrm{H} 460$ cells that had been treated with 0.2 and $0.4 \mathrm{mM}$ escitalopram oxalate for $24 \mathrm{~h}$, relative to those in the control group (Fig. 5).

Signaling molecules involved in the escitalopram oxalate induced-apoptosis in A549 and H460 cells. To identify signaling pathways that may be involved in escitalopram oxalate-induced apoptosis in A549 and H460 cells, the expressions of $\mathrm{p}-\mathrm{I} \kappa \mathrm{B}-\alpha$ and NF- $\kappa \mathrm{B}$ (p65-p) proteins were examined. The expressions of both $\mathrm{p}-\mathrm{I} \kappa \mathrm{B}-\alpha$ and $\mathrm{NF}-\kappa \mathrm{B}(\mathrm{p} 65-\mathrm{p})$ proteins were significantly increased in both A549 and H460 cells following treatment with 0.2 and $0.4 \mathrm{mM}$ escitalopram oxalate for $24 \mathrm{~h}$, relative to those in the control group (Fig. 6).

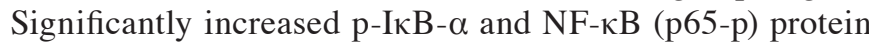
levels were also detected in $\mathrm{H} 460$ cells that had been treated with at 0.2 and $0.4 \mathrm{mM}$ escitalopram oxalate for $24 \mathrm{~h}$, relative to those in the control group (Fig. 6).

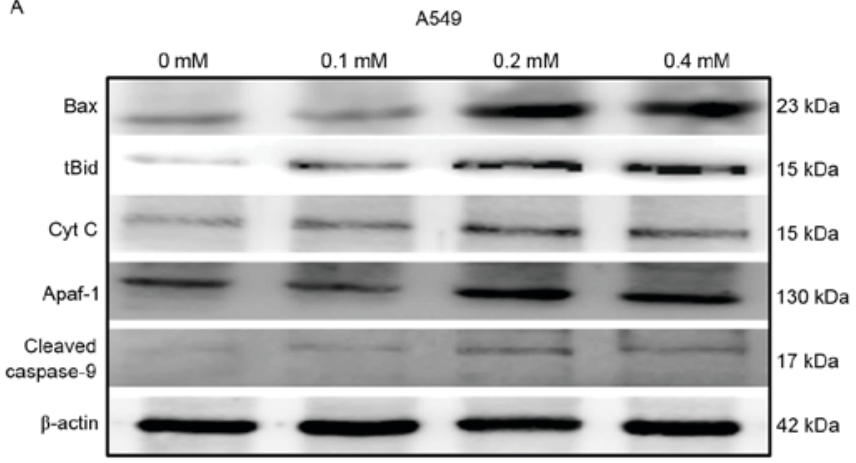

B

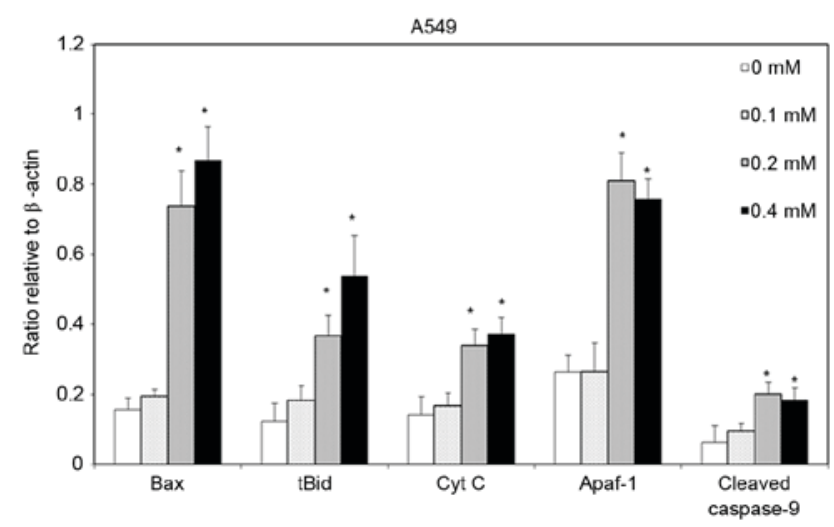

Figure 4. Escitalopram oxalate induced mitochondrial dependent apoptosis in A549 cells. (A) The expressions of Bax, tBid, cytochrome $c$, Apaf-1 and cleaved caspase-9 proteins in A549 cells that were treated with different concentration of escitalopram oxalate for $24 \mathrm{~h}$ were detected by western blotting. (B) Bars represent the relative protein levels of Bax, tBid, cytochrome $c$, Apaf- 1 and cleaved caspase- 9 proteins on the basis of $\beta$-actin. ${ }^{*} \mathrm{P}<0.05$ was considered to indicate a significant difference, as compared to the control group $(0 \mathrm{mM})$. Similar results were observed in three repeated experiments.

\section{Discussion}

Neuroleptic medications are reportedly associated with a reduced risk of certain cancers. However, relatively little is known about the mechanism by which SSRI causes NSCLC cell death. This study demonstrated that escitalopram oxalate, a more effective SSRI, has significantly inhibitory effects on the proliferation and invasive ability of A549 and H460 cells, relative to the controls. Escitalopram oxalate also caused significant apoptosis by inducing mitochondria-associated cascades in both A549 and H460 cells. These findings suggest that escitalopram oxalate may have therapeutic potential against NSCLC.

Cell migration is known to involve a complex mechanism. It is required for many biological activities, including embryogenesis, wound healing, immune response and tissue repair (13). Errors in the cell migration process cause serious pathologic episodes, including cancer invasion and metastasis $(14,15)$, which are important characteristics of malignant tumor cells (16). Cancer metastasis comprises four essential steps, including detachment, migration, invasion and adhesion, which are different but interrelated $(17,18)$. Once cancer cells have spread beyond their initial primary site, the cancer is typically incurable and fatal (19). 


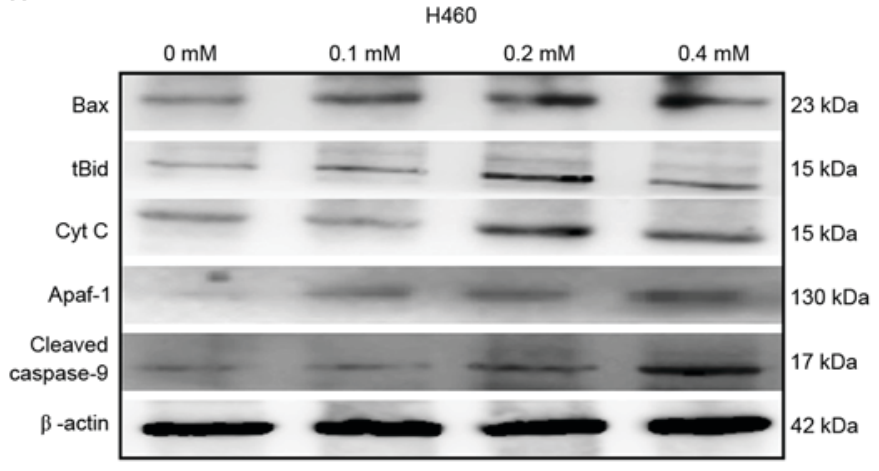

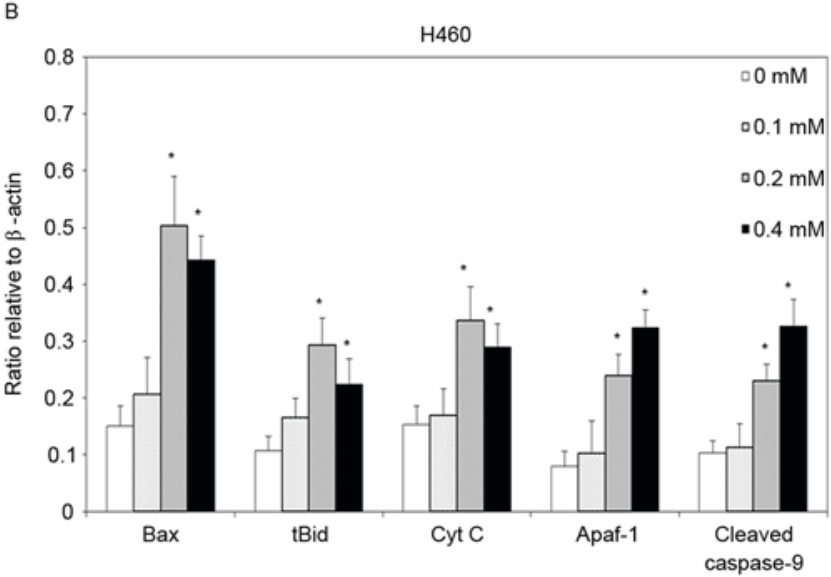

Figure 5. Escitalopram oxalate induced mitochondrial dependent apoptosis in H460 cells. (A) The expressions of Bax, tBid, cytochrome $c$, Apaf-1 and cleaved caspase-9 proteins in $\mathrm{H} 460$ cells that were treated with different concentration of escitalopram oxalate for $24 \mathrm{~h}$ were detected by western blotting. (B) Bars represent the relative protein levels of Bax, tBid, cytochrome $c$, Apaf- 1 and cleaved caspase- 9 proteins on the basis of $\beta$-actin. " $\mathrm{P}<0.05$ was considered to indicate a significant difference, as compared to the control group $(0 \mathrm{mM})$. Similar results were observed in three repeated experiments.

A
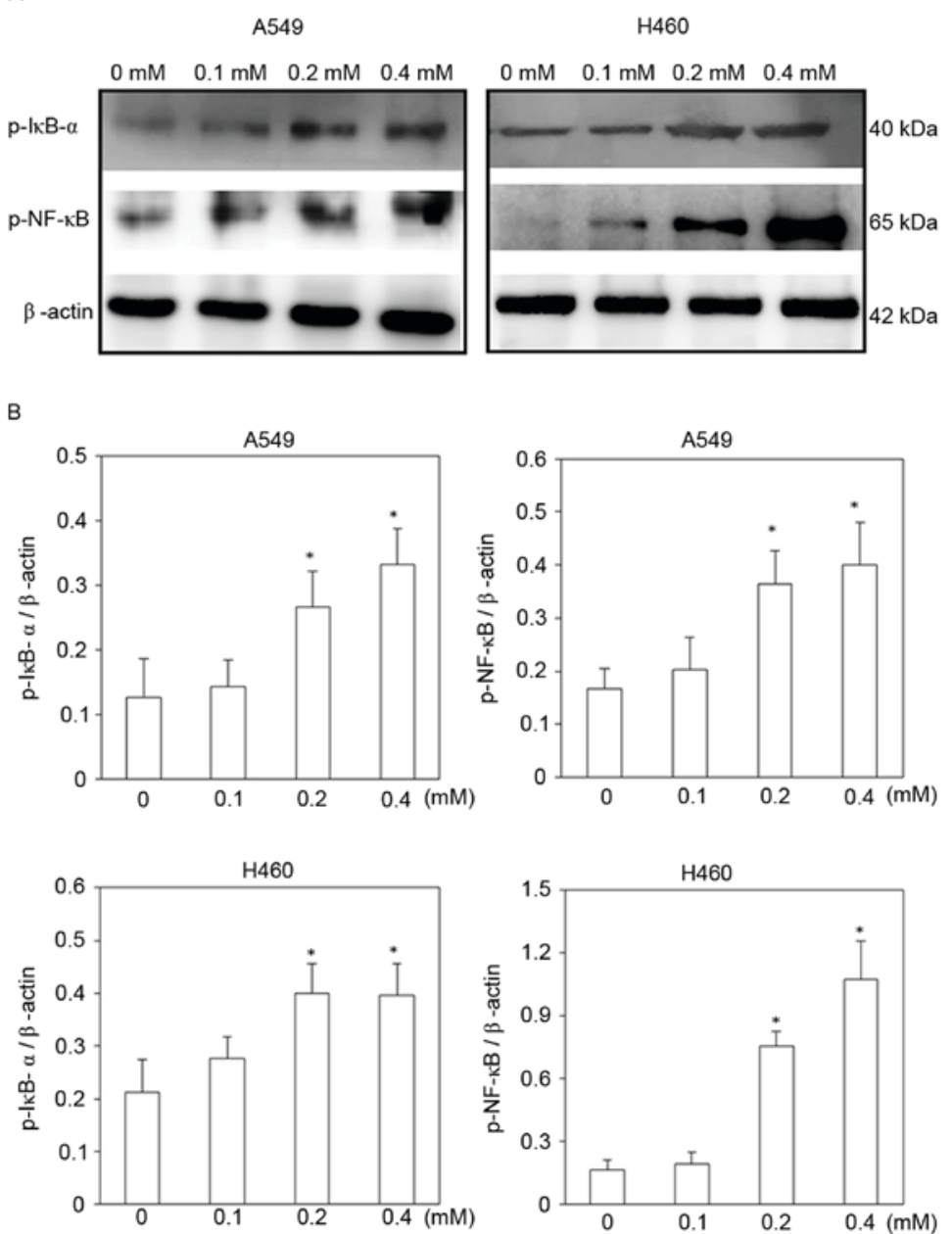

Figure 6. Escitalopram oxalate induces the expressions of p-IкB- $\alpha$ and NF-кB (p65-p) proteins in A549 and H460 cells. (A) The expressions of p-IкB- $\alpha$ and NF- $\mathrm{kB}(\mathrm{p} 65-\mathrm{p})$ proteins in A549 and H460 cells that were treated with different concentrations of escitalopram oxalate for $24 \mathrm{~h}$ were detected by western blotting. (B) Bars represent the relative protein levels of Bax, tBid, cytochrome $c$, Apaf- 1 and cleaved caspase- 9 proteins on the basis of $\beta$-actin. " $\mathrm{P}<0.05$ was considered to indicate a significant difference, as compared to the control group $(0 \mathrm{mM})$. Similar results were observed in three repeated experiments. $\mathrm{p}$-IкB- $\alpha$, phosphorylated IкB- $\alpha$.

Cancer metastasis is the leading cause of morbidity and mortality, and is responsible for almost $90 \%$ of all cancer mortality (20). Therefore, constraining cancer metastasis is important in cancer therapy. In this study, escitalopram 
oxalate significantly reduced the motility and invasive abilities of A549 and H460 cells, exhibiting a potential to inhibit the migration of NSCLC cells.

Unlimited proliferation is known to be a critical process in cell carcinogenesis. Therefore, compounds that suppress tumor growth by inducing cell cycle arrest and apoptosis are favored for cancer therapy $(21,22)$. The induction of apoptosis is one of the main mechanisms for impeding cancer growth and is regarded as necessary for screening novel anti-cancer agents (23). The mechanisms of apoptosis are highly complex and sophisticated. The two main pathways of apoptosis are extrinsic and intrinsic, which are linked with each other (24). The extrinsic signaling pathways that initiate apoptosis involve transmembrane receptor-mediated interactions, such as the engagement of tumor necrosis factor (TNF) and TNF receptor (25). The intrinsic signaling pathways that induce apoptosis involve intracellular signals that act directly on targets in the cell and are mitochondria-initiated events. The events involve cytochrome c, which activates the caspase-dependent mitochondrial pathway. Cytochrome $c$ binds and activates Apaf-1 and procaspase-9, forming an 'apoptosome' (26). The clustering of procaspase- 9 in this manner eventually results in caspase- 9 and caspase- 3 activation (27). In this study, the cytotoxic activity of escitalopram oxalate was triggered by releasing Bax, tBid, cytochrome $c$ and Apaf-1, resulting in the proteolytic cleavage of caspase- 9 and caspase- 3 in A549 and H460 cells. These findings suggest that the therapeutic efficacy of escitalopram oxalate against NSCLC cells involves inducing mitochondria-dependent apoptosis. However, other possible mechanisms and interactive targets that are involved in escitalopram oxalate-induced cell death in NSCLC cells warrant further investigation.

Evidence reveals that neuroleptic medications are associated with a reduced cancer risk $(6,28)$. Various SSRIs, especially fluoxetine, are known to reduce the risk of cancer (29-31), including lung cancer (9). However, the side-effects of fluoxetine remain problematic (32). Escitalopram oxalate is a superior SSRI that has been demonstrated to have favorable tolerability and to cause generally milder and more temporary adverse events than other SSRIs (11). The present study firstly demonstrated that escitalopram oxalate significantly inhibits the proliferation and invasion of A549 and H460 cells and induces mitochondria-dependent apoptosis therein. These findings suggest that escitalopram oxalate is more effective than other SSRIs and probably effective for reducing the risk of NSCLC development.

In summary, this study firstly revealed that escitalopram oxalate significantly inhibits the viability and mobility in A549 and H460 cells, resulting in subsequent mitochondria-dependent apoptosis through $\mathrm{p}-\mathrm{I} \kappa \mathrm{B}-\alpha / \mathrm{NF}-\kappa \mathrm{B}$ (p65-p) signaling. Accordingly, escitalopram oxalate also reduces cell viability and mobility and induces apoptosis in the control cell lines, BEAS-2B; however, these phenomena are milder than those detected in A549 and H460 cells. Although further animal study may be required, this study reveals that escitalopram oxalate exhibits significant cytotoxic effects on A549 and H460 cells and suggests a therapeutic potential of escitalopram oxalate in the treatment of NSCLC patients.

\section{Acknowledgements}

This work was supported by clinical research grant from Kaohsiung Armed Force General Hospital, Kaohsiung, Taiwan (no. 105-30) and partially supported by Chang Gung Medical Foundation, Chiayi Chang Gung Memorial Hospital and Chang Gung University, Chiayi, Taiwan-Taiwan Ministry of Health and Welfare Clinical Trial and Research Center of Excellence (CMRPG6E0272). Ted Knoy is appreciated for his editorial assistance.

\section{References}

1. Islami F, Torre LA and Jemal A: Global trends of lung cancer mortality and smoking prevalence. Transl Lung Cancer Res 4: 327-338, 2015.

2. Torre LA, Siegel RL and Jemal A: Lung cancer statistics. Adv Exp Med Biol 893: 1-19, 2016.

3. Petersen I and Warth A: Lung cancer: Developments, concepts, and specific aspects of the new WHO classification. J Cancer Res Clin Oncol 142: 895-904, 2016.

4. Herbst RS, Heymach JV and Lippman SM: Lung cancer. N Engl J Med 359: 1367-1380, 2008.

5. American Cancer Society: Lung Cancer (Non-Small Cell): American Joint Committee on Cancer Staging (AJCC-2010). Atlanta, GA, 2013.

6. Jones GR: Cancer therapy: Phenothiazines in an unexpected role. Tumori 71: 563-569, 1985.

7. Toh S, Rodríguez LA and Hernández-Díaz S: Use of antidepressants and risk of lung cancer. Cancer Causes Control 18: 1055-1064, 2007

8. Dodd S, Berk M, Kelin K, Zhang Q, Eriksson E, Deberdt W and Craig Nelson J: Application of the Gradient Boosted method in randomised clinical trials: Participant variables that contribute to depression treatment efficacy of duloxetine, SSRIs or placebo. J Affect Disord 168: 284-293, 2014.

9. Stepulak A, Rzeski W, Sifringer M, Brocke K, Gratopp A, Kupisz K, Turski L and Ikonomidou C: Fluoxetine inhibits the extracellular signal regulated kinase pathway and suppresses growth of cancer cells. Cancer Biol Ther 7: 1685-1693, 2008.

10. Burke WJ: Escitalopram. Expert Opin Investig Drugs 11: 1477-1486, 2002.

11. Kirino E: Escitalopram for the management of major depressive disorder: A review of its efficacy, safety and patient acceptability. Patient Prefer Adherence 6: 853-861, 2012.

12. Chiu CC, Shi YF, Yang JJ, Hsiao YC, Tzang BS and Hsu TC: Effects of human parvovirus B19 and bocavirus VP1 unique region on tight junction of human airway epithelial A549 cells. PLoS One 9: e107970, 2014.

13. Binamé F, Pawlak G, Roux P and Hibner U: What makes cells move: Requirements and obstacles for spontaneous cell motility. Mol Biosyst 6: 648-661, 2010.

14. Friedl $\mathrm{P}$ and Wolf K: Tumour-cell invasion and migration: Diversity and escape mechanisms. Nat Rev Cancer 3: 362-374, 2003.

15. Sahai E: Mechanisms of cancer cell invasion. Curr Opin Genet Dev 15: 87-96, 2005

16. Liotta LA, Rao CN and Wewer UM: Biochemical interactions of tumor cells with the basement membrane. Ann Rev Biochem 55: 1037-1057, 1986.

17. Gabbert H: Mechanisms of tumor invasion: Evidence from in vivo observations. Cancer Metastasis Rev 4: 293-309, 1985.

18. Guan X: Cancer metastases: Challenges and opportunities. Acta Pharm Sin B 5: 402-418, 2015.

19. Wells A, Grahovac J, Wheeler S, Ma B and Lauffenburger D: Targeting tumor cell motility as a strategy against invasion and metastasis. Trends Pharmacol Sci 34: 283-289, 2013.

20. Seyfried TN and Huysentruyt LC: On the origin of cancer metastasis. Crit Rev Oncog 18: 43-73, 2013.

21. Los M, Burek CJ, Stroh C, Benedyk K, Hug H and Mackiewicz A: Anticancer drugs of tomorrow: Apoptotic pathways as target for drug design. Drug Discov Today 8: 67-77, 2003.

22. Qiu J, Zhao BB, Shen Y, Chen W, Ma YD and Shen YM: A novel p-terphenyl derivative inducing cell-cycle arrest and apoptosis in MDA-MB-435 cells through topoisomerase inhibition. Eur J Med Chem 68: 192-202, 2013. 
23. Wong RS: Apoptosis in cancer: From pathogenesis to treatment. J Exp Clin Cancer Res 30: 87, 2011.

24. Igney FH and Krammer PH: Death and anti-death: Tumour resistance to apoptosis. Nat Rev Cancer 2: 277-288, 2002.

25. Locksley RM, Killeen N and Lenardo MJ: The TNF and TNF receptor superfamilies: Integrating mammalian biology. Cell 104: 487-501, 2001.

26. Hill MM, Adrain C, Duriez PJ, Creagh EM and Martin SJ: Analysis of the composition, assembly kinetics and activity of native Apaf-1 apoptosomes. EMBO J 23: 2134-2145, 2004.

27. Elmore S: Apoptosis: A review of programmed cell death. Toxicol Pathol 35: 495-516, 2007.

28. Dalton SO, Johansen C, Poulsen AH, Nørgaard M, Sørensen HT, McLaughlin JK, Mortensen PB and Friis S: Cancer risk among users of neuroleptic medication: A population-based cohort study. Br J Cancer 95: 934-939, 2006.

29. Mun AR, Lee SJ, Kim GB, Kang HS, Kim JS and Kim SJ: Fluoxetine-induced apoptosis in hepatocellular carcinoma cells. Anticancer Res 33: 3691-3697, 2013.
30. Kraft SL, Baker NM, Carpenter J and Bostwick JR: Procarbazine and antidepressants: A retrospective review of the risk of serotonin toxicity. Psychooncology 23: 108-113, 2014.

31. Kannen V, Garcia SB, Silva WA Jr, Gasser M, Mönch R, Alho EJ, Heinsen H, Scholz CJ, Friedrich M, Heinze KG, et al: Oncostatic effects of fluoxetine in experimental colon cancer models. Cell Signal 27: 1781-1788, 2015.

32. Andersen J, Kristensen AS, Bang-Andersen B and Strømgaard K Recent advances in the understanding of the interaction of antidepressant drugs with serotonin and norepinephrine transporters. Chem Commun (Camb) 25: 3677-3692, 2009. 